\title{
Comparative genomics reveals insights into avian genome evolution and adaptation
}

Guojie Zhang, ${ }^{1,2 *}+$ Cai Li, ${ }^{1,3 *}$ Qiye Li, ${ }^{1,3}$ Bo Li, ${ }^{1}$ Denis M. Larkin, ${ }^{4}$ Chul Lee, ${ }^{5,6}$ Jay F. Storz, ${ }^{7}$ Agostinho Antunes, ${ }^{8,9}$ Matthew J. Greenwold, ${ }^{10}$ Robert W. Meredith, ${ }^{11}$ Anders Ödeen, ${ }^{12}$ Jie Cui, ${ }^{13,14}$ Qi Zhou, ${ }^{15}$ Luohao Xu, ${ }^{1,16}$ Hailin Pan, ${ }^{1}$ Zongji Wang, ${ }^{1,17}$ Lijun Jin, ${ }^{1}$ Pei Zhang, ${ }^{1}$ Haofu Hu, ${ }^{1}$ Wei Yang, ${ }^{1}$ Jiang Hu, ${ }^{1}$ Jin Xiao, ${ }^{1}$ Zhikai Yang, Yang Liu, ${ }^{1}$ Qiaolin Xie, ${ }^{1}$ Hao Yu, ${ }^{1}$ Jinmin Lian, ${ }^{1}$ Ping Wen, ${ }^{1}$ Fang Zhang, ${ }^{1}$ Hui Li, ${ }^{1}$ Yongli Zeng, ${ }^{1}$ Zijun Xiong, ${ }^{1}$ Shiping Liu, ${ }^{1,17}$ Long Zhou, ${ }^{1}$ Zhiyong Huang, ${ }^{1}$ Na An, Jie Wang, ${ }^{1,18}$ Qiumei Zheng, ${ }^{1}$ Yingqi Xiong, ${ }^{1}$ Guangbiao Wang, ${ }^{1}$ Bo Wang, Jingjing Wang, ${ }^{1}$ Yu Fan, ${ }^{19}$ Rute R. da Fonseca, ${ }^{3}$ Alonzo Alfaro-Núñez, ${ }^{3}$ Mikkel Schubert, ${ }^{3}$ Ludovic Orlando, ${ }^{3}$ Tobias Mourier, ${ }^{3}$ Jason T. Howard, ${ }^{20}$ Ganeshkumar Ganapathy, ${ }^{20}$ Andreas Pfenning, ${ }^{20}$ Osceola Whitney, ${ }^{20}$ Miriam V. Rivas, ${ }^{20}$ Erina Hara, ${ }^{20}$ Julia Smith, ${ }^{20}$ Marta Farré, ${ }^{4}$ Jitendra Narayan, ${ }^{21}$ Gancho Slavov, ${ }^{21}$ Michael N Romanov, ${ }^{22}$ Rui Borges, ${ }^{8,9}$ João Paulo Machado, ${ }^{8,23}$ Imran Khan, ${ }^{8,9}$ Mark S. Springer, ${ }^{24}$ John Gatesy, ${ }^{24}$ Federico G. Hoffmann, ${ }^{25,26}$ Juan C. Opazo, ${ }^{27}$ Olle Håstad, ${ }^{28}$ Roger H. Sawyer, ${ }^{10}$ Heebal Kim, ${ }^{5,6,29}$ Kyu-Won Kim, ${ }^{5}$ Hyeon Jeong Kim, ${ }^{6}$ Seoae Cho, ${ }^{6}$ Ning $\mathrm{Li}^{30}{ }^{30}$ Yinhua Huang, ${ }^{30,31}$ Michael W. Bruford, ${ }^{32}$ Xiangjiang Zhan, ${ }^{32,33}$ Andrew Dixon, ${ }^{34}$ Mads F. Bertelsen, ${ }^{35}$ Elizabeth Derryberry, ${ }^{36,37}$ Wesley Warren, ${ }^{38}$ Richard K Wilson, ${ }^{38}$ Shengbin Li, ${ }^{39}$ David A. Ray, ${ }^{26} \neq$ Richard E. Green, ${ }^{40}$ Stephen J. O'Brien, ${ }^{41,42}$ Darren Griffin, ${ }^{22}$ Warren E. Johnson, ${ }^{43}$ David Haussler, ${ }^{40}$ Oliver A. Ryder, ${ }^{44}$ Eske Willerslev, ${ }^{3}$ Gary R. Graves, ${ }^{45,46}$ Per Alström, ${ }^{47,48}$ Jon Fjeldså, ${ }^{46}$ David P. Mindell, ${ }^{49}$ Scott V. Edwards, ${ }^{50}$ Edward L. Braun, ${ }^{51}$ Carsten Rahbek, 46,52 David W. Burt, ${ }^{53}$ Peter Houde, ${ }^{54}$ Yong Zhang, Huanming Yang, ${ }^{1,55}$ Jian Wang, ${ }^{1}$ Avian Genome Consortium, $\S$ Erich D. Jarvis, ${ }^{20}+$ M. Thomas P. Gilbert, ${ }^{3,56}+$ Jun Wang ${ }^{1,55,57,58,59}+$

Birds are the most species-rich class of tetrapod vertebrates and have wide relevance across many research fields. We explored bird macroevolution using full genomes from 48 avian species representing all major extant clades. The avian genome is principally characterized by its constrained size, which predominantly arose because of lineage-specific erosion of repetitive elements, large segmental deletions, and gene loss. Avian genomes furthermore show a remarkably high degree of evolutionary stasis at the levels of nucleotide sequence, gene synteny, and chromosomal structure. Despite this pattern of conservation, we detected many non-neutral evolutionary changes in protein-coding genes and noncoding regions. These analyses reveal that pan-avian genomic diversity covaries with adaptations to different lifestyles and convergent evolution of traits.

W ith 10,500 living species (1), birds are the most species-rich class of tetrapod vertebrates. Birds originated from a theropod lineage more than 150 million years ago during the Jurassic and are the only extant descendants of dinosaurs $(2,3)$. The earliest diversification of extant birds (Neornithes) occurred during the Cretaceous period. However, the Neoaves, the most diverse avian clade, later underwent a rapid global expansion and radiation after a mass extinction event $\sim 66$ million years ago near the Cretaceous-Paleogene (K-Pg) boundary $(4,5)$. As a result, the extant avian lineages exhibit extremely diverse morphologies and rates of diversification. Given the nearly complete global inventory of avian species, and the immense collected amount of distributional and biological data, birds are widely used as models for investigating evolutionary and ecological ques- tions $(6,7)$. The chicken (Gallus gallus), zebra finch (Taeniopygia guttata), and pigeon (rock dove) (Columba livia) are also important model organisms in disciplines such as neuroscience and developmental biology (8). In addition, birds are widely used for global conservation priorities (9) and are culturally important to human somesticated and are economically important. Farmed and wild water birds are key players in the global spread of pathogens, such as avian influenza virus (10).

Despite the need to better understand avian genomics, annotated avian genomic data was previously available for only a few species: the domestic chicken, domestic turkey (Meleagris gallopavo) and zebra finch (11-13), together with a few others only published recently (14-16). To build an understanding of the genetic complexcieties. A number of avian species have been do- ity of birds and to investigate links between their genomic variation and phenotypic diversity, we collected and compared genome sequences of these and other avian species (48 species total), representing all 32 neognath and two of the five palaeognath orders (Fig. 1) (17), thus representing nearly all of the major clades of living birds (5).

\section{Results}

\section{Sequencing, assembly, and annotation}

We used a whole-genome shotgun strategy to generate genome sequences of 45 new avian species (18), including two species representing two orders within the infraclass Paleognathae [common ostrich (Struthio camelus) and whitethroated tinamou (Tinamus guttatus)], the other order within Galloanserae [Peking duck (Anas platyrhynchos)], and 41 species representing 30 neoavian orders (table S1) (19). In combination with the three previously published avian genomes (11-13), the genome assemblies cover $92 \%$ (34 of 37) of all avian orders (the three missing orders belong to the Paleognathae) (17). With the exception of the budgerigar (Melopsittacus undulatus), which was assembled through a multiplatform (Illumina/GS-FLX/PacBio) approach (20), all other new genomes were sequenced and assembled with Illumina (San Diego, CA) short reads (Fig. 1) (18). For 20 species, we produced high $(>50 \times)$ coverage sequences from multiple libraries, with a gradient of insert sizes and built full-genome assemblies. For the remaining 25 species, we generated low $(\sim 30 \times)$ coverage data from two insert-size libraries and built less complete but still sufficient assemblies for comparative genome analyses. These de novo (18) genome assemblies ranged from 1.05 to $1.26 \mathrm{~Gb}$, which is consistent with estimated cytology-based genome sizes (21), suggesting near complete genome coverage for all species. Scaffold N50 sizes for high-coverage genomes ranged from 1.2 to $6.9 \mathrm{Mb}$, whereas those for lowercoverage genomes were $\sim 48 \mathrm{~kb}$ on average (table S2). The genomes of the ostrich and budgerigar were further assembled with optical maps, increasing their scaffold N50 sizes to 17.7 and $13.8 \mathrm{Mb}$, respectively $(20,22)$.

We annotated the protein-coding sequences using a homology-based method for all genomes, aided by transcriptome sequencing for some species (18). To avoid systematic biases related to the use of different methods in annotations of previously published avian genomes, we created a uniform reference gene set that included all genes from the chicken, zebra finch, and human (23). This database was used to predict protein gene models in all avian genomes and American alligator (Alligator mississippiensis) (24). All high-coverage genomes were predicted to contain $\sim 15,000$ to 16,000 transposable element-free protein-coding genes [table S3 and annotation files in (19)], similar to the chicken genome $(\sim 15,000)$. Despite the fragmented nature of the lowcoverage genomes leading to $\sim 3000$ genes likely missing or partially annotated, it was still possible to predict 70 to $80 \%$ of the entire catalog of avian genes. 


\section{Broad patterns of avian genome evolution}

Although many fishes and some amphibians have smaller genomes than birds, among amniotes, birds have the smallest (21). The genomes of mammals and nonavian reptiles typically range from 1.0 to $8.2 \mathrm{~Gb}$, whereas avian genomes range from 0.91 in the black-chinned hummingbird (Archilochus alexanderi) to a little over $1.3 \mathrm{~Gb}$ in the common ostrich (21). A number of hypotheses have been proposed for the smaller avian genome size (25-28). Here, we document key events that have likely contributed to this smaller genome size.

The proliferation and loss of transposable elements (TEs) may drive vertebrate genome size evolution (29-31). Consistent with the zebra finch and galliformes genomes (11-13, 32), almost all avian genomes contained lower levels of repeat elements ( $\sim$ to $10 \%$ of each genome) (table S4) than in other tetrapod vertebrates (for example, 34 to $52 \%$ in mammals) (33). The sole outlier was the downy woodpecker (Picoides pubescens), with TEs representing $\sim 22 \%$ of the genome, derived mainly from species-specific expansion of LINE (long interspersed elements) type CR1 (chicken repeat 1) transposons (fig. S1). In contrast, the average total length of SINEs (short interspersed elements) in birds has been reduced to $\sim 1.3 \mathrm{Mb}$, which is $\sim 10$ to 27 times less than in other reptiles [12.6 $\mathrm{Mb}$ in alligator; $34.9 \mathrm{Mb}$ in green sea turtle (Chelonia mydas)], suggesting that a deficiency of SINEs occurred in the common ancestor of birds.

We compared the average size of genomic elements of birds with 24 mammalian and the three nonavian reptile genomes. Avian protein-coding genes were on average 50 and $27 \%$ shorter than the mammalian and reptilian genes, respectively (Fig. 2A). This reduction is largely due to the shortening of introns and reduced intergenic distances that resulted in an increased gene density (Fig. 2A). Such genomic contraction has also evolved convergently in bats (fig. S11), the only flying mammalian group. The condensed genomes may represent an adaptation tied to rapid gene regulation required during powered flight $(34,35)$.

To further investigate whether avian genome size reduction is due to a lineage-specific reduction in the common avian ancestor of birds or expansion in other vertebrates (36), we performed ancestral state reconstructions of small $[<100$ base pairs (bp)] deletion events across an alignment of four representative well-assembled avian and three reptile genomes (18) and found that the avian ancestral lineage experienced the largest number of small deletion events-about twice the number in the common ancestor of birds and crocodiles (fig. S12). In contrast, many fewer small deletion events occurred in modern avian lineages (fig. S12).

We next created a gene synteny map between the highest-quality assembled avian genome (ostrich) and other reptile genomes to document lineage-specific events of large segmental deletions (18). We detected 118 syntenic blocks, spanning a total of $58 \mathrm{Mb}$, that are present in alligator and turtle genomes but lost in all birds (table S8). In contrast, $\sim 8 \mathrm{x}$ and $\sim 5 \mathrm{x}$ fewer syntentic blocks were missing in alligator (14 blocks, $9 \mathrm{Mb}$ ) and turtle (27 blocks, $8 \mathrm{Mb}$ ) relative to green anole, respectively, confirming the polarity of genome size reduction in birds (table S8). The large segmental losses in birds were skewed to losses from chr2 and chr6 of the green anole (fig. S13). Two of the green anole's 12 pairs of microchromosomes, LGd and LGf, were completely missing in birds, with no homologous genes found within the avian genomes. Most of these lost segments were located at the ends of chromosomes or close to the centrosomes (fig. S13). Furthermore, lost segments were enriched at apparent breakpoints of the avian microchromosomes (Fig. 2B and fig. S13). These findings imply that the large segmen tal losses may be a consequence of chromosomal fragmentation events in the common ancestor of birds giving rise to additional microchromosomes in modern birds.

The large segmental deletions in birds contain at least 1241 functional protein-coding genes (table S9), with each lost segment containing at least five contiguous genes. The largest region lost in birds was a 2.1-Mb segment of the green anole chr2, which contains 28 protein-coding genes (Fig. 2B). Overall, at least $7 \%$ of the green anole macrochromosomal genes were lost through segmental deletions in birds. Although gene loss is a common evolutionary process, this massive level of segmental deletion has not been previously observed in vertebrates. Over $77 \%$ of the 1241 genes present in the large segmentally deleted regions have at least one additional paralog in the green anole genome, a level higher than the overall percentage of genes with paralogs in the green anole genome or avian genomes (both at $\sim 70 \%$ ). This suggests that birds may

${ }^{1}$ China National GeneBank, Beijing Genomics Institute (BGI)-Shenzhen, Shenzhen, 518083, China. ${ }^{2}$ Centre for Social Evolution, Department of Biology, Universitetsparken 15, University of Copenhagen, DK-2100 Copenhagen, Denmark. ${ }^{3}$ Centre for GeoGenetics, Natural History Museum of Denmark, University of Copenhagen, Øster Voldgade 5-7, 1350 Copenhagen, Denmark. ${ }^{4}$ Royal Veterinary College, University of London, London, UK. ${ }^{5}$ Interdisciplinary Program in Bioinformatics, Seoul National University, Seoul 151-742, Republic of Korea. ${ }^{6}$ Cho and Kim Genomics, Seoul National University Research Park, Seoul 151-919, Republic of Korea. ' School of Biological Sciences, University of Nebraska, Lincoln, NE 68588, USA. ${ }^{8}$ Centro de Investigación en Ciencias del Mar y Limnología (CIMAR)/Centro Interdisciplinar de Investigação Marinha e Ambiental (CIIMAR), Universidade do Porto, Rua dos Bragas, 177, 4050-123 Porto, Portugal. ${ }^{9}$ Departamento de Biologia, Faculdade de Ciências, Universidade do Porto, Rua do Campo Alegre, 4169-007 Porto, Portugal. ${ }^{10}$ Department of Biological Sciences, University of South Carolina, Columbia, SC, USA. ${ }^{11}$ Department of Biology and Molecular Biology, Montclair State University, Montclair, NJ 07043, USA. ${ }^{12}$ Department of Animal Ecology, Uppsala University, Norbyvägen 18D, S-752 36 Uppsala, Sweden. ${ }^{13}$ Marie Bashir Institute for Infectious Diseases and Biosecurity, Charles Perkins Centre, School of Biological Sciences and Sydney Medical School, The University of Sydney, Sydney, NSW 2006, Australia. ${ }^{14}$ Program in Emerging Infectious Diseases, Duke-NUS Graduate Medical School, Singapore 169857. Singapore. ${ }^{15}$ Department of Integrative Biology University of California, Berkeley, CA 94720, USA. ${ }^{16}$ College of Life Sciences, Wuhan University, Wuhan 430072, China. ${ }^{17}$ School of Bioscience and Bioengineering, South China University of Technology, Guangzhou 510006, China. ${ }^{18}$ BGl Education Center,University of Chinese Academy of Sciences, Shenzhen, 518083, China. ${ }^{19}$ Key Laboratory of Animal Models and Human Disease Mechanisms of Chinese Academy of Sciences and Yunnan Province, Kunming Institute of Zoology, Kunming, Yunnan 650223. China. ${ }^{20}$ Department of Neurobiology, Howard Hughes Medical Institute. Duke University Medical Center. Durham, NC 27710, USA. ${ }^{21}$ Institute of Biological, Environmental and Rural Sciences, Aberystwyth University, Aberystwyth, UK. ${ }^{22}$ School of Biosciences, University of Kent, Canterbury CT2 7NJ, UK. ${ }^{23}$ Instituto de Ciências Biomédicas Abel Salazar (ICBAS), Universidade do Porto, Portugal. ${ }^{24}$ Department of Biology, University of California Riverside, Riverside, CA 92521, USA. ${ }^{25}$ Department of Biochemistry, Molecular Biology, Entomology and Plant Pathology, Mississippi State University, Mississippi State, MS 39762, USA. ${ }^{26}$ Institute for Genomics, Biocomputing and Biotechnology. Mississippi State University. Mississippi State, MS 39762, USA. ${ }^{27}$ Instituto de Ciencias Ambientales y Evolutivas, Facultad de Ciencias, Universidad Austral de Chile, Valdivia, Chile. ${ }^{28}$ Department of Anatomy, Physiology and Biochemistry, Swedish University of Agricultural Sciences, Post Office Box 7011, S-750 07, Uppsala, Sweden. ${ }^{29}$ Department of Agricultural Biotechnology and Research Institute for Agriculture and Life Sciences, Seoul National University, Seoul 151-742, Republic of Korea. ${ }^{30}$ State Key Laboratory for Agrobiotechnology, China Agricultural University, Beijing 100094, China. ${ }^{31} \mathrm{College}$ of Animal Science and Technology, China Agricultural University, Beijing 100094, China. ${ }^{32}$ Organisms and Environment Division, Cardiff School of Biosciences, Cardiff University, Cardiff CF10 3AX, Wales, UK. ${ }^{33}$ Key Lab of Animal Ecology and Conservation Biology, Institute of Zoology, Chinese Academy of Sciences, Beijing 100101 China. ${ }^{34}$ International Wildlife Consultants, Carmarthen SA33 5YL, Wales, UK. ${ }^{35}$ Centre for Zoo and Wild Animal Health, Copenhagen Zoo, Roskildevej 38 , DK-2000 Frederiksberg Denmark. ${ }^{36}$ Department of Ecology and Evolutionary Biology, Tulane University, New Orleans, LA, USA. ${ }^{37}$ Museum of Natural Science, Louisiana State University, Baton Rouge, LA 70803, USA. ${ }^{38 T h e}$ Genome Institute at Washington University, St. Louis, MO 63108, USA. ${ }^{39}$ College of Medicine and Forensics, Xi'an Jiaotong University, Xi'an, 710061 , China. ${ }^{40}$ Department of Biomolecular Engineering, University of California, Santa Cruz, CA 95064, USA. ${ }^{41}$ Theodosius Dobzhansky Center for Genome Bioinformatics, St. Petersburg State University, St. Petersburg, Russia. ${ }^{42}$ Nova Southeastern University Oceanographic Center 8000 N Ocean Drive, Dania, FL 33004, USA. ${ }^{43}$ Smithsonian Conservation Biology Institute, National Zoological Park, 1500 Remount Road, Front Royal, VA 22630, USA. ${ }^{44}$ Genetics Division, San Diego Zoo Institute for Conservation Research, 15600 San Pasqual Valley Road, Escondido, CA 92027, USA. ${ }^{45}$ Department of Vertebrate Zoology, MRC-116, National Museum of Natural History, Smithsonian Institution, Post Office Box 37012, Washington, DC 20013-7012, USA. ${ }^{46} \mathrm{Center}$ for Macroecology, Evolution and Climate, the Natural History Museum of Denmark, University of Copenhagen, Universitetsparken 15, DK-2100 Copenhagen 0, Denmark. ${ }^{47}$ Key Laboratory of Zoological Systematics and Evolution, Institute of Zoology, Chinese Academy of Sciences, 1 Beichen West Road, Chaoyang District, Beijing 100101, China. ${ }^{48}$ Swedish Species Information Centre, Swedish University of Agricultural Sciences, Box 7007, SE-750 07 Uppsala, Sweden. ${ }^{49}$ Department of Biochemistry \& Biophysics, University of California, San Francisco, CA 94158, USA. ${ }^{50}$ Department of Organismic and Evolutionary Biology and Museum of Comparative Zoology, Harvard University, 26 Oxford Street, Cambridge, MA 02138, USA. ${ }^{51}$ Department of Biology and Genetics Institute, University of Florida, Gainesville, FL 32611, USA. ${ }^{52}$ Imperial College London, Grand Challenges in Ecosystems and the Environment Initiative, Silwood Park Campus, Ascot, Berkshire SL5 7PY, UK. ${ }^{53}$ Division of Genetics and Genomics, The Roslin Institute and Royal (Dick) School of Veterinary Studies, The Roslin Institute Building, University of Edinburgh, Easter Bush Campus, Midlothian EH25 9RG, UK. ${ }^{54}$ Department of Biology, New Mexico State University, Box 30001 MSC 3 AF, Las Cruces, NM 88003 , USA. ${ }^{55}$ Macau University of Science and Technology, Avenida Wai long, Taipa, Macau 999078, China. ${ }^{56}$ Trace and Environmental DNA Laboratory, Department of Environment and Agriculture, Curtin University, Perth, Western Australia, 6102, Australia. ${ }^{5}$ Department of Biology, University of Copenhagen, Ole Maaløes Vej 5, 2200 Copenhagen, Denmark. ${ }^{58}$ Princess Al Jawhara Center of Excellence in the Research of Hereditary Disorders, King Abdulaziz University, Jeddah 21589, Saudi Arabia. ${ }^{59}$ Department of Medicine, University of Hong Kong, Hong Kong.

*These authors contributed equally to this work. †Corresponding author. E-mail: zhanggj@genomics.cn (G.Z.); jarvis@neuro.duke.edu (E.D.J.); mtpgilbert@gmail.com (M.T.P.G.); wangj@genomics.cn (J.W.) łPresent address: Department of Biological Sciences, Texas Tech University, Lubbock, TX 79409, USA. §Avian Genome Consortium authors and affiliations are listed at the end of this paper. 
have undergone functional compensation in their paralogous gene copies, reducing selection against the loss of these segmental regions. We predict that the loss of functions associated with many genes in the avian ancestor may have had a profound influence on avian-specific traits (table S11).

\section{Conservative mode of genome evolution}

With $\sim 2 / 3$ of avian species possessing $\sim 30$ pairs of microchromosomes, the avian karyotype appears to be distinctly conserved because this phenotype is not a general feature of any other vertebrate group studied to date (37). We assessed the rates of avian chromosomal evolution among the 21 more fully assembled genomes (scaffold N50 > 1 Mb) (table S2) (18). From the alignment of chicken with the other 20 avian genomes, plus green anole and Boa constrictor (38), we identified homologous synteny blocks (HSBs) and 1746 evolutionary breakpoint regions (EBRs) in different avian lineages and then estimated the expected number of EBRs (18) and the rates of genomic rearrangements, using a phylogenetic total evidence nucleotide tree (TENT) as a guide (5). We excluded the turkey genome after detecting an unusually high fraction of small lineagespecific rearrangements, suggesting a high number of local misassemblies. Of the 18 remaining nonSanger-sequenced genomes (table S2), the estimated rate of chimeric scaffolds that could lead to false EBRs was 6\% (39).

The average rate of rearrangements in birds is $\sim 1.25$ EBRs per million years; however, bursts of

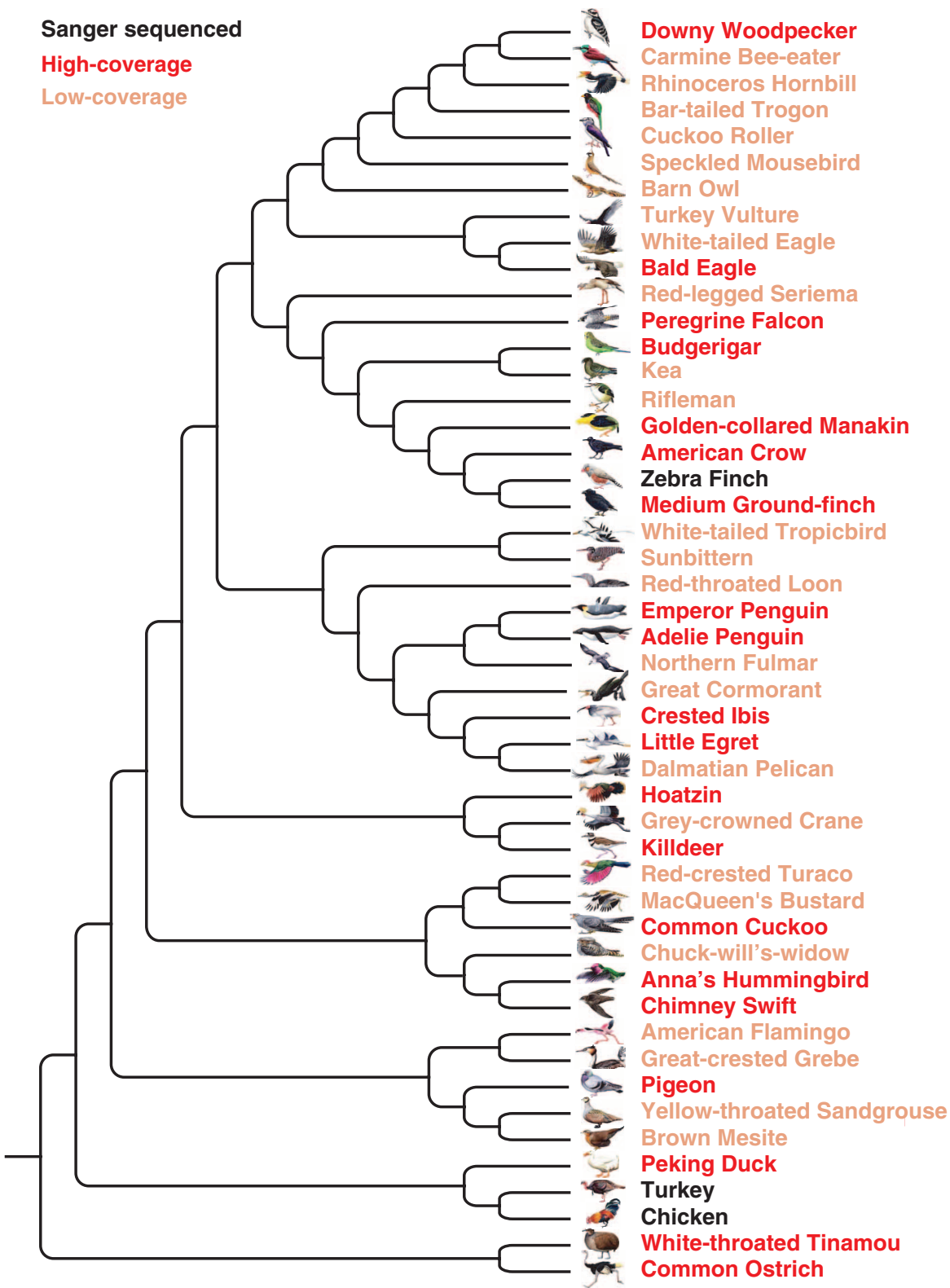

Fig. 1. Avian family tree and genomes sequenced. The phylogenomic relationships of the 48 avian genomes from (5), with Sanger-sequenced (black), high-coverage (dark red), and low-coverage (light red) genomes denoted. genomic reorganization occurred in several avian lineages (fig. S15). For example, the origin of Neognathae was accompanied by an elevated rate of chromosome rearrangements ( 2.87 EBRs per million years). Intriguingly, all vocal learning species [zebra finch, medium-ground finch (Geospiza fortis), American crow (Corvus brachyrhynchos), budgerigar, and Anna's hummingbird (Calypte anna)] had significantly higher rates of rearrangements than those of close vocal nonlearning relatives [golden-collared manakin (Manacus vitellinus), peregrine falcon (Falco peregrinus) and chimney swift (Chaetura pelagica)] [phylogenetic analysis of variance $F$ statistic $(F)=5.78, P=0.0499]$ and even higher relative to all vocal nonlearning species $(F=$ 15.03, $P=0.004)$. This may be related to the larger radiations these clades experienced relative to most other bird groups. However, the golden-collared manakin, which belongs to suboscines (vocal nonlearners) that have undergone a larger radiation than parrots and hummingbirds, has a low rearrangement rate.

We next compared microsynteny (local gene arrangements), which is more robust and accurate than macrosynteny analyses for draft assemblies (18). We compared with eutherian mammals, which are approximately the same evolutionary age as Neoaves and whose genome assemblies are of similar quality. We examined the fraction of orthologous genes identified from each pair of two-avian/mammalian genomes, on the basis of syntenic and best reciprocal blast matches (18). Birds have a significantly higher percentage of synteny-defined orthologous genes than that of mammals (Fig. 2C). The fraction of genes retained in syntenic blocks in any pairwise comparison was linearly related with evolutionary time, by which the overall level of genome shuffling in birds was lower than in mammals over the past $\sim 100$ million years (Fig. $2 \mathrm{C}$ ). This suggests a higher level of constraint on maintaining gene synteny in birds relative to mammals.

The apparent stasis in avian chromosome evolution suggests that birds may have experienced relatively low rates of gene gain and loss in multigene families. We examined the intensively studied gene families that encode the various $\alpha$ - and $\beta$-type subunits of hemoglobin, the tetrameric protein responsible for blood oxygen transport in jawed vertebrates (40). In amniotes, the $\alpha$ - and $\beta$-globin gene families are located on different chromosomes (40) and experienced high rates of gene turnover because of lineage-specific duplication and deletion events (41). In birds, the size and membership composition of the globin gene families have remained remarkably constant during 100 million years of evolution, with most examined species retaining an identical complement (Fig. 2D). Estimated gene turnover rates $(\lambda)$ of $\alpha$ - and $\beta$-globin gene families were over twofold higher in mammals than birds ( $\lambda=0.0023$ versus 0.0011 , respectively). Much of the variation in the avian $\alpha$-globin gene family was attributable to multiple independent inactivations of the $\alpha D$-globin gene (Fig. $2 D$ ), which encodes the $\alpha$-chain subunit of a 
A
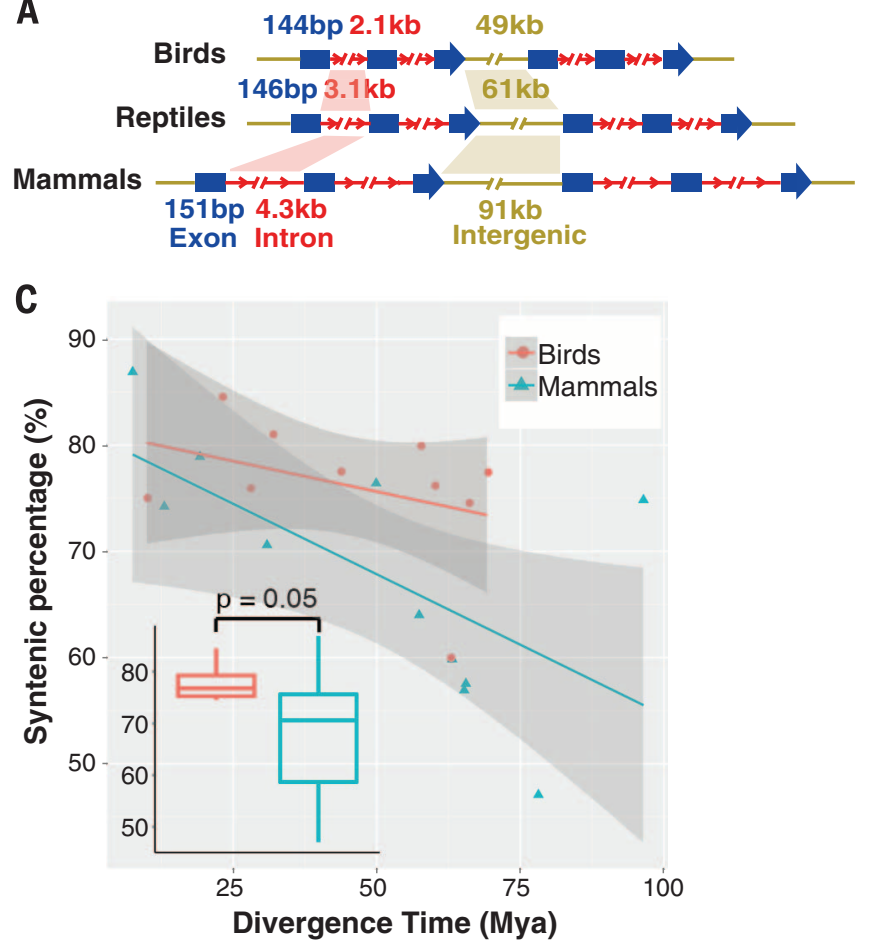

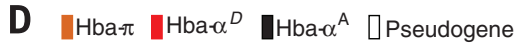

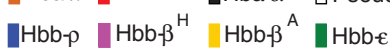

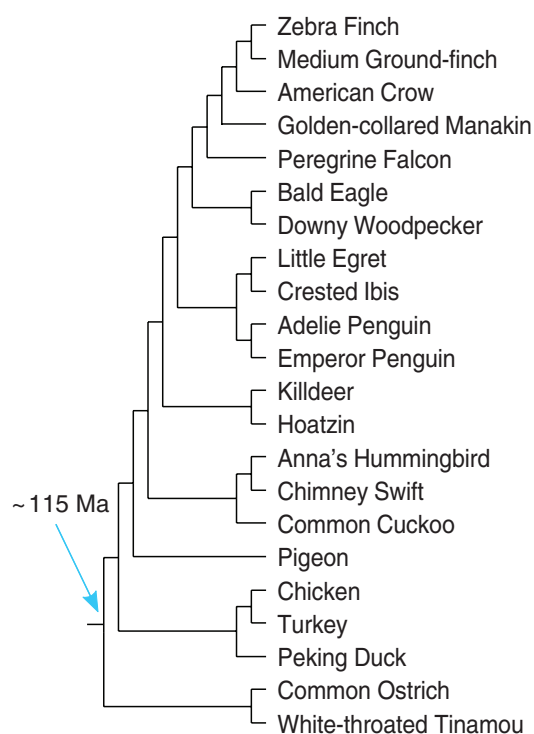

B

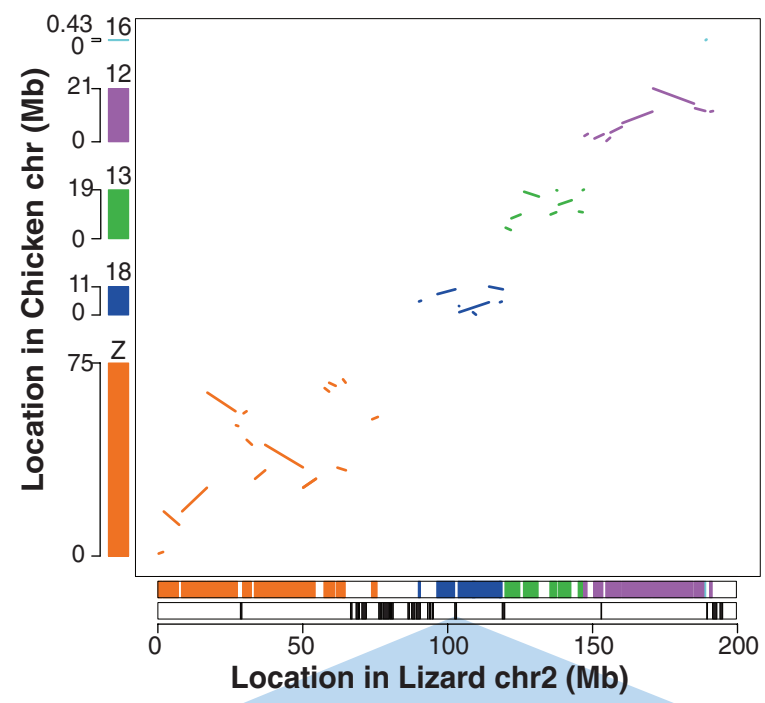

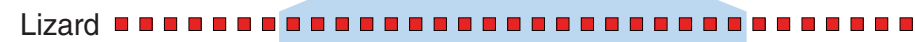
Turtle

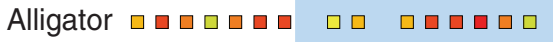
Birds $\square \square \square \quad \square \otimes$

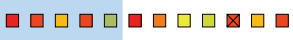
\ $\quad \square \quad \square \square \square \square \otimes \otimes \square \square$ 0 $50 \quad 100$ identity pseudo

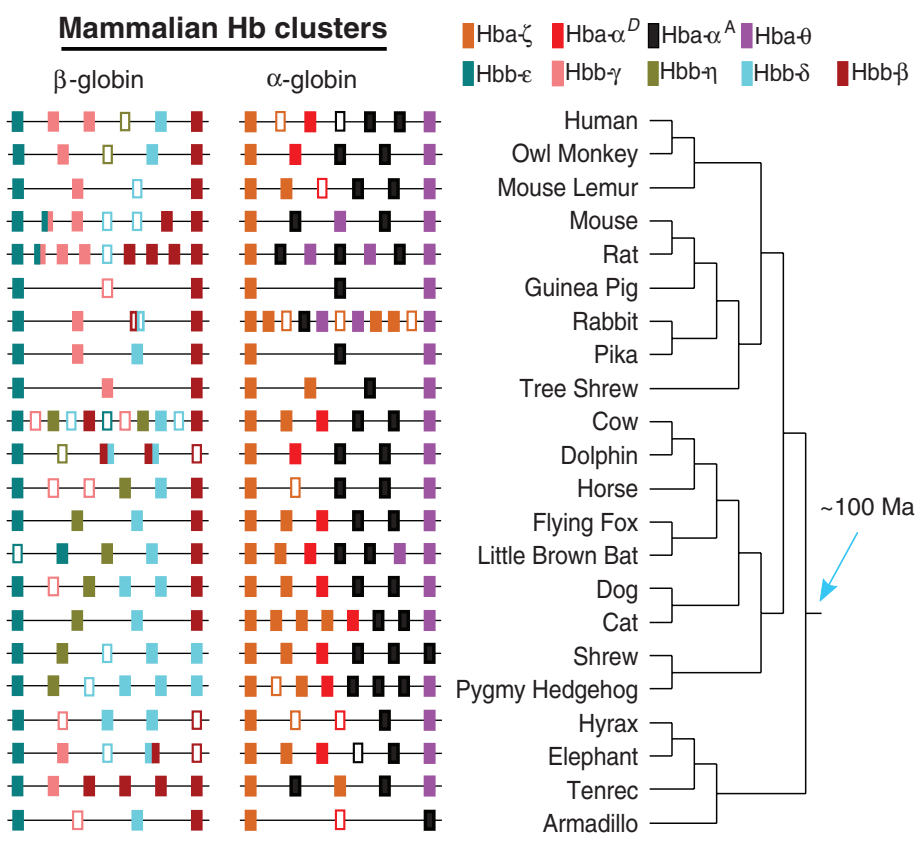

pairwise comparisons between two avian or mammalian species. Box plots indicate that the overall distributions of the synteny percentages in birds and mammals are different ( $P$ value was calculated by using Wilcoxon rank sum test with phylogenetically independent species pairs). (D) Chromosomal organization of the $\alpha$ - and $\beta$-globin gene clusters in representative avian and mammalian taxa. These genes encode the $\alpha$ - and $\beta$-type subunits of tetrameric ( $\alpha 2 \beta 2)$ hemoglobin isoforms that are expressed at different ontogenetic stages. In the case of the $\alpha$-like globin genes, birds and mammals share orthologous copies of the $\alpha . D$ - and $\alpha A$-globin genes. Likewise, the avian $\pi$ globin and the mammalian $\zeta$-globin genes are 1:1 orthologs. In contrast, the genes in the avian and mammalian $\beta$-globin gene clusters are derived from independent duplications of one or more $\beta$-like globin genes that were inherited from the common ancestor of tetrapod vertebrates $(90,91)$. 
hemoglobin isoform ( $\mathrm{HbD})$ expressed in both embryonic and definitive erythrocytes (42). Because of uniform and consistent differences in oxygen-binding properties between $\mathrm{HbD}$ and the major adult-expressed hemoglobin isoform, $\mathrm{HbA}$ (which incorporates products of $\alpha A$-globin) (42), the inactivations of $\alpha D$-globin likely contribute to variation in blood-oxygen affinity, which has important consequences for circulatory oxygen transport and aerobic energy metabolism. Overall, the globin gene families illustrate a general pattern of evolutionary stasis in birds relative to mammals.

Genomic nucleotide substitution rates vary across species and are determined through both neutral and adaptive evolutionary processes (43). We found that the overall pan-genomic background substitution rate in birds $\left(\sim 1.9 \times 10^{-3}\right.$ substitutions per site per million years) was lower than in mammals $\left(\sim 2.7 \times 10^{-3}\right.$ substitution per site per million years) (Fig. 3A). However, the substitution rate estimates also exhibited interordinal variation among birds (Fig. 3A). There was a positive correlation between the substitution rate and the number of species per order [coefficient of determination $\left(R^{2}\right)=0.21, P=0.01$, Pearson's test with phylogenetically independent contrasts] (Fig. 3B and fig. S19), evidencing an association with rates of macroevolution (44). For example, Passeriformes, the most diverse avian order, exhibited the highest evolutionary rate $\left(\sim 3.3 \times 10^{-3}\right.$ substitutions per site per million years), almost two times the average of Neoaves $\left(\sim 2 \times 10^{-3}\right.$ substitutions per site per million years, Fig. 3A). Landbirds exhibited an average higher substitution rate than that of waterbirds (landbirds, $\sim 2.2 \times 10^{-3}$ substitutions per site per million years; waterbirds, $\sim 1.6 \times 10^{-3}$ substitutions per site per million years), which is consistent with the observation that landbirds have greater net diversification rates than those of waterbirds (7). Among the landbirds, the predatory lineages exhibited slower rates of evolution $\left(\sim 1.6 \times 10^{-3}\right.$ substitutions per site per million years), similar to that of waterbirds. Moreover, the three vocal learning landbird lineages (parrots, songbirds, and hummingbirds) are evolving faster than are nonvocal learners (Fig. 3A). Overall, our analyses indicate that genome-wide variation in rates of substitution is a consequence of the avian radiation into a wide range of niches and associated phenotypic changes.

\section{Selective constraints on functional elements}

Conservation of DNA sequences across distantly related species reflects functional constraints (45). A direct comparison of $100-\mathrm{Mb}$ orthologous genomic regions revealed more regions evolving slower than the neutral rate among birds (Fig. 3C) than mammals (46), which is consistent with the slower rate of avian mitochondrial sequence evolution (47). We predicted 3.2 million highly conserved elements (HCEs) at a resolution of $10 \mathrm{bp}$ or greater spanning on average $7.5 \%$ of the avian genome, suggesting a strong functional constraint in avian genomes. Functional annotations revealed that $\sim 12.6 \%$ of these HCEs were associated with protein-coding genes, whereas
A
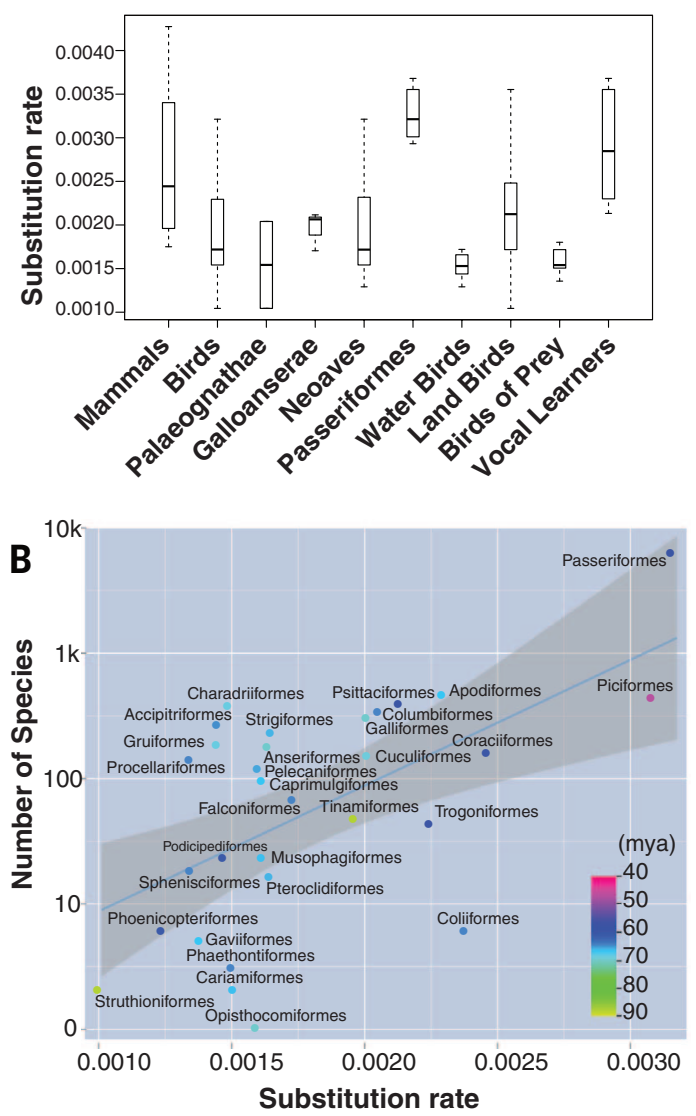

Fig. 3. Evolutionary rate and selection constraints. (A) Substitution rate in each lineage was estimated by the comparison of fourfold degenerate (4d) sites in coding regions, in units of substitutions per site per million years. Waterbirds and landbirds are defined in (5). (B) Correlation between average substitution rates and number of species within different avian orders. Divergence times were estimates from (5). The fit line was derived from least square regression analysis, and the confidence interval was estimated by "stat_smooth" in R. The units of the $x$ axis are numbers of substitutions per site per million years. The correlation figure with phylogenetically indepen-
D

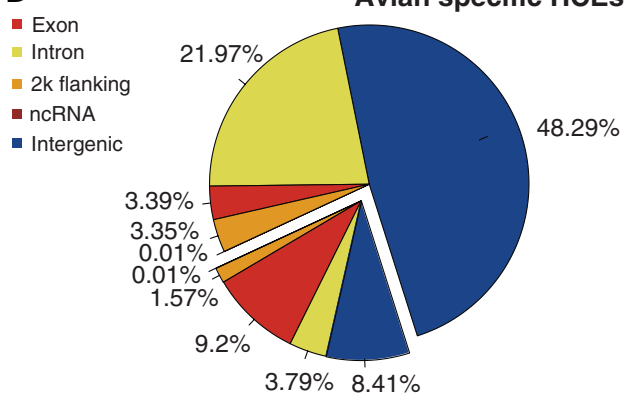

HCEs shared by birds and mammals
E

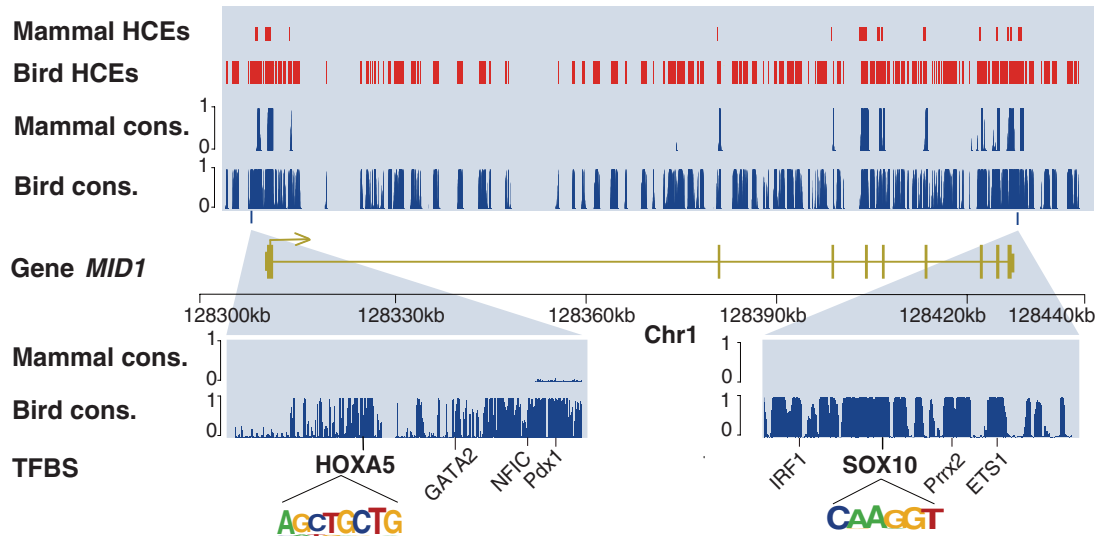

dent contrasts is provided in the supplementary materials. (C) Density map for comparison of conservation levels between pan-avian and pan-mammalian genomes, on the basis of the homologous genomic regions between birds and mammals. Conservation levels were quantified by means of PhastCons basewise conservation scores. (D) HCEs found in both mammalian and avian genomes (smaller pie piece) and those that are avian-specific (larger pie piece). (E) MID1 contains abundant avian-specific HCEs in the upstream and downstream regulatory regions. Many regulatory motif elements are identified in these avian-specific HCEs. Cons., conservation level. 
the majority of the remaining HCEs were located in intron and intergenic regions (Fig. 3, D and E). These HCEs enabled us to identify 717 new proteincoding exons and 137 new protein-coding genes, with $77 \%$ of the latter supported by the deep transcriptome data (table S17). Deep transcriptome sequencing also enabled us to annotate 5879 candidate long noncoding RNA (lncRNA) genes, of which 220 overlapped HCEs with a coverage ratio of $>50 \%$ (table S18) (18).

Because HCEs may have different functions in different lineages, we separated the HCEs into two categories: bird-specific and amniote HCEs (shared by birds and mammals). Among the birdspecific HCEs, we identified 13 protein-coding genes that were highly conserved in birds but divergent in mammals (table S19). One of the most conserved was the sperm adhesion gene, SPAMI, which mediates sperm binding to the egg coat (48). This gene, however, was under positive selection driven by sperm competition in mammalian species (49). Noncoding HCEs play important roles in the regulation of gene expression (50); thus, we compared the transcription factor binding sites in the ENCODE project (51) with the HCEs and found that the avian-specific HCEs are significantly associated with transcription factors functioning in metabolism (table S20), whereas amniote core HCEs are enriched with transcription factors functioning in signal regulation, stimulus responses, and development (table S21).

To investigate evolutionary constraints on gene regions, we calculated $\mathrm{d} N / \mathrm{d} S$ [the ratio of the number of nonsynonymous substitutions per nonsynonymous site $(\mathrm{dN})$ to the number of synonymous substitutions per synonymous site $(\mathrm{d} S)$ ] for 8295 high-quality orthologs. Consistent with the fast-Z sex chromosome hypothesis (52), the evolutionary rate of Z-linked genes was significantly higher than autosome genes (Fig. 4A). This is most likely driven by the reduction of effective population size $\left(\mathrm{N}_{\mathrm{e}}\right)$ of Z-linked genesbecause the $\mathrm{N}_{\mathrm{e}}$ of $\mathrm{Z}$ chromosome is only $3 / 4$ of that of autosomes-as well as by male sexual selection (52). Furthermore, consistent with the fast-macro hypothesis, the overall rate of macro- chromosomal genic evolution is higher than that of microchromosomes (Fig. 4A), which is probably due to differences in the recombination rates and genic densities between macro- and microchromosomes in birds (53).

We also examined the $\mathrm{d} N / \mathrm{d} S$ ratio of each avian Gene Ontology (GO) category for cominvolved in development (such as spinal cord development and bone resorption) are evolving faster in birds, and those involved in the brain function (such as synapse assembly, synaptic vesicle transport, and neural crest cell migration) are evolving faster in mammals (tables S23 and S24). Genes involved in oxidoreductase activity were relatively rapidly evolving in the Palaeognathae clade that contains the flightless ratites (Fig. 4B and table S25). The fast evolving GOs in the Galloanserae participate in regulatory functions (Fig. 4B and table S26). In Neoaves, genes parison with mammals and within birds. Those

involved in microtubule-based processes were the fastest evolving (Fig. 4B and table S27). We speculate that these differences could be caused by relaxed selective constraints or positive selection in different lineages.

\section{Genotype-phenotype convergent associations: Evolution of vocal learning}

With the availability of genomes representing all major modern avian lineages and their revised phylogenetic relationships (5), it becomes possible to conduct genome-wide association studies across species with convergent traits. We focused on vocal learning, which given our phylogenetic analyses is inferred as having evolved independently, either twice, in hummingbirds and the common ancestor of songbirds and parrots, or three times $(5,54)$. All three groups have specialized song-learning forebrain circuits (song nuclei) not found in vocal nonlearners (Fig. 5A) (55).

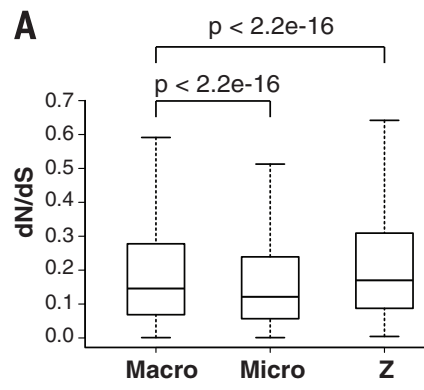

Fig. 4. Selection constraints on genes. (A) Box plot for the distribution of $\mathrm{d} N / \mathrm{d} S$ values of genes on avian macrochromosomes, microchromosomes, and the Z chromosome. $P$ values were calculated with Wilcoxon rank sum tests. (B) GO categories in Neoaves, Galloanserae, and Palaeognathae showing clade-specific rapid evolutionary rates. Red bars, $P$ value of significance; blue bars, number of genes in each GO.

B maintenance of location
chll cycle process
chromosome segregation
transmembrane transport
establishment of localization
regulation of biological process
regulation of cellular process
regulation of signaling regulation

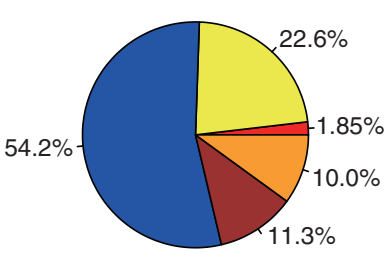

- Exon B

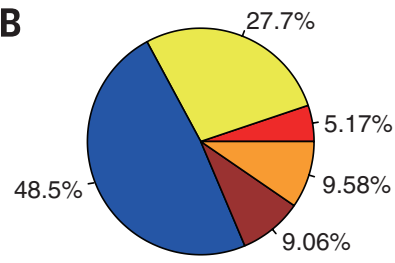

Multiple sequence alignment
Intron - Intergenic - 5' $10 \mathrm{k}$ 3' 10k
Fig. 5. Convergent molecular changes among vocal learning birds. (A) Songbird brain diagram showing the specialized forebrain song-learning nuclei (yellow) that controls the production (HVC and RA) and acquisition (LMAN and Area X) of learned song (55).

Gray arrows indicate connections between brain regions; red and blue (thick) arrows in-

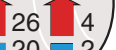
dicate relative numbers of genes with increased or decreased specialized expression in zebra finch song Thalamus nuclei and with convergent accelerated coding sequences (left numbers of 66 total) or convergent amino RA, robust nucleus of the arcopallium; LMAN, lateral magnocellular nucleus of the anterior nidopallium; Area X, Area X of the striatum; and HVC, a letter-based name. (B) Classification of vocal learner-specific accelerated elements, compared with the background alignment of 15 avian species. 
Analyses of 7909 orthologous protein-coding genes with available amino acid sites in all three vocal-learning and control vocal nonlearning groups revealed convergent accelerated $\mathrm{d} N / \mathrm{d} S$ for 227 genes in vocal learners (table S28). Of these, $73 \%$ (165) were expressed in the songbird brain (physically cloned mRNAs), and of these, $92 \%$ (151) were expressed in adult song-learning nuclei, which is much higher than the expected $60 \%$ of brain genes expressed in song nuclei (56). About $20 \%$ (33) were regulated by singing, which is twice the expected $10 \%(56)$. In addition, $41 \%$ of the song nuclei accelerated genes showed differential expression among song nuclei [expected $20 \%(56)$ ], and 0.7 to $9 \%$ [0.7 to $4.3 \%$ expected (57)] showed specialized expression compared with the surrounding brain regions (table S28) (58). GO analyses of the accelerated differentially expressed song nuclei genes revealed 30 significant functionally enriched gene sets, which clustered into four major categories, including neural connectivity, brain development, and neural metabolism (fig. S25). For an independent measure of convergence, we developed an approach that scans for single amino acid substitutions common to species with a shared trait, controlling for phylogenetic relationships (18). Of the 7909 genes, 38 had one to two amino acid substitutions present only in vocal learners (table S31). At least $66 \%$ of these were expressed in the songbird brain, including in the song nuclei [58\%; 20\% expected (56)]. Two genes (GDPD4 and KLAA1919) showed convergent accelerated evolution on the amino acid sites specific to vocal learners (table S31).

To identify accelerated evolution in noncoding sequences in vocal learners, we scanned the genome alignment using phyloP $(18,59)$. We used a more limited sampling of vocal nonlearning species closely related to the vocal learners (table S32) because of the relatively faster evolutionary rate of noncoding regions. We scanned the entire genome alignment and found 822 accelerated genomic elements specifically shared by all three vocal learning groups (table S33). These convergent elements were skewed to intergenic regions in vocal learners relative to the background average accelerated elements across species (Fisher's exact test, $P<2.2 \times 10^{-16}$ ) (Fig. 5B). Of these elements, 332 were associated with 278 genes (within $10 \mathrm{~kb} 5^{\prime}$ or $3^{\prime}$ of the nearest gene), of which a high proportion (76\%) was expressed in the brain; almost all of those (94\%, 198 genes) expressed in one or more song nuclei, $20 \%$ were regulated by singing ( $10 \%$ expected), $51 \%$ ( $20 \%$ expected) showed differential expression among song nuclei, and 2 to $15 \%$ [0.7 to $4.3 \%$ expected, based on (56)] had specialized expression relative to the surrounding brain regions, including the FoxP1 gene involved in speech (table S34 and figs. S27 to S32). Overall, these analyses show a 2- to 3.5-fold enrichment of accelerated evolution in regulatory regions of genes differentially expressed in vocal learning brain regions. In contrast, there was very little overlap (2.5\%) of genes with convergent accelerated noncoding changes and convergent accelerated amino acid changes, indicating two independent targets of selection for convergent evolution.

\section{Evolution of ecologically relevant genes}

We also investigated candidate genes that underlie traits relevant to avian ecological diversity. Although these analyses should be approached with caution given the phenotypic and ecological plasticity within major avian lineages, we examined genes putatively associated with major skeletal and tissue changes for the capacity for powered flight, feeding modification such as loss of teeth, the advanced visual system found in some lineages, and sexual and reproductive systems.

\section{Evolution of the capacity for flight}

Skeletal systems: The evolution of flight involved a series of adaptive changes at the morphological and molecular levels. One of the key requirements for flight is a skeleton that is both strong and lightweight. In both birds and nonavian theropods, this evolved through the fusion and elimination of some bones and the pneumatization of the remaining ones (60). Of 89 genes involved in ossification (table S36), 49 ( $55 \%$ ) showed evidence of positive selection in birds, which is almost twice as high as in mammals (31 genes, $\sim 35 \%$ ). For birds, most of these are involved in the regulation of remodeling and ossification-associated processes, or bone development in general, and those with the highest values for global $\mathrm{d} N / \mathrm{d} S(>0.5)$ were obtained for AHSG ( $\alpha-2-H S-g l y c o p r o t e i n)$, which is associated with bone mineral density, and $P 2 R X 7$ ( $P 2 X$ purinoceptor 7 ), which is associated with bone homeostasis. The variation in the extension of pneumatization in avian post-cranial bones has been associated with the variation in body size and foraging strategies (61). Therefore, selection of these genes may explain variation in the levels of bone pneumatization in birds because the genes involved in the process of maintaining trabeculae within bones likely depends on the intrinsic network of genes participating in bone resorption and mineralization. These results suggest that most structural differences in bone between birds and mammals may be a result of bone remodeling and resorption (table S37).

Pulmonary structure and function: The increased metabolism associated with homeothermy and powered flight requires an efficient gas exchange process during pulmonary ventilation. Because of functional integration of ventilation and locomotion, birds evolved a volume-constant lung and a rigid trunk region, whereas mammals evolved a changing-volume lung, often coupled to locomotory flexion of the lumbar region (62). In contrast to the pulmonary alveola of the mammalian lung, the avian lung has a honeycomb-like structure incorporating a flow-through system with small air capillaries (63). We found five genes that function in mammalian lung development that were lost in the avian ancestor (table S11).

Feathers: The evolution and subsequent morphological diversification of feathers have shaped avian physiology, locomotion, mate choice, and ecological niches (64). Feathers are composed of $\alpha$ - and $\beta$-keratins (65), the latter of which are structural proteins found only in the epidermal appendages of birds and other reptiles. The $\alpha$-keratin gene family has contracted in birds relative to reptiles (except turtle) and mammals (0.7-fold change), whereas the $\beta$-keratin gene family has expanded (1.96-fold change) relative to reptiles (Fig. 6A and table S39). The avian $\beta$-keratins form six clusters, with all major avian lineages possessing members from each avian cluster (fig. S33), indicating that avian $\beta$-keratin diversity was present in the basal avian lineage. Of these, the feather $\beta$-keratin subfamily is avian-specific and comprises over $56 \%$ of the genes, whereas the remaining avian $\beta$-keratin subfamilies (claw, scale, and keratinocyte $\beta$-keratin subfamilies) are found in turtles and crocodiles (Fig. 6A and fig. S33). The mean number of keratinocyte $\beta$-keratins is similar across bird groups and their two closest living reptile relatives (turtle and alligator), suggesting copy number conservation since their common ancestor (Fig. 6A). In contrast, aquatic/semi-aquatic birds have a relatively low mean number of feather $\beta$-keratins compared with that of land birds, with land birds having more than double the number, and among them several domesticated land birds (zebra finch, chicken, pigeon, and budgerigar) having more than 8 times (Fig. 6A). Although the later observation is concordant with the hypothesis that domestication may increase the recombination rate at $\beta$-keratin loci $(66$, 67), domestic turkey and Peking duck did not exhibit this trend. Overall, these findings indicate that feather compositional adaptations are associated with different avian lifestyles.

\section{Evolution of genes related to diet}

Edentulism: The evolution of birds also had major consequences with regard to their feeding strategies and diets, with changes at the structural, biochemical, and sensory levels (among others). One of the most immediately obvious avian-specific traits is edentulism, the phenotype of being toothless. Edentulism is thought to have evolved independently in multiple theropod lineages (68). However, although most phylogenetic analyses suggest that teeth were lost in the common ancestor of modern birds (69), several studies have recovered dentate taxa (Hesperornis and Ichthyornis) from the Mesozoic inside of crown Neornithes, suggesting that tooth loss could have occurred independently (70). A scan of avian genomes for molecular fossils of tooth-specific genes recovered remnants of enamel and dentin formation genes in all species examined [table 1 in (71)]. Frameshift mutations and whole-exon deletions were widespread in all investigated tooth genes. The vast majority of debilitating mutations were not shared, but all species shared unambiguous deletions in protein-coding exons of enamel-specific genes (ENAM, AMEL, AMBN, $M M P 20$, and $A M T N$ ) and one dentin-specific gene $(D S P P)$. This shared pattern of pseudogenization across living birds supports the hypothesis that the common ancestor of modern birds lacked mineralized teeth (69). 
Diet-related enzymes: Birds have evolved an extraordinary diversity of dietary specializations. The glyoxylate detoxifying enzyme alanine/glyoxylate aminotransferase (AGT) represents a candidate for study (72). We recovered complete AGT genes from 22 avian genomes (table S42), of which five exhibit pseudogenized forms in their MTS region (Fig. 6B and fig. S34). MTS function was lost in three unrelated avian orders, which is consistent with multiple independent dietary transitions during avian evolution. Detection of positively selected amino acids at $137 \mathrm{Q}(\mathrm{dN} / \mathrm{d} S=2.153)$ and $378 \mathrm{R}$ $(\mathrm{d} N / \mathrm{d} S=2.153)$ in all birds provided additional support for diet-related adaptation in AGT (positions according to human AGT; posterior probability $>99 \% ; P<0.0001)$.

Vitamin $\mathrm{C}(\mathrm{Vc})$ is an important nutrient cofactor in a range of essential metabolic reactions. Loss of the ability to synthesize Vc has occurred in humans, Guinea pigs, and some bats. All species that do not synthesize Vc exhibit a pseudogenized gene for L-gulonolactone oxidase (GULO), an enzyme essential for catalyzing the last step of Vc synthesis (73). Genomic mining revealed GULO pseudogenization in two oscines (medium
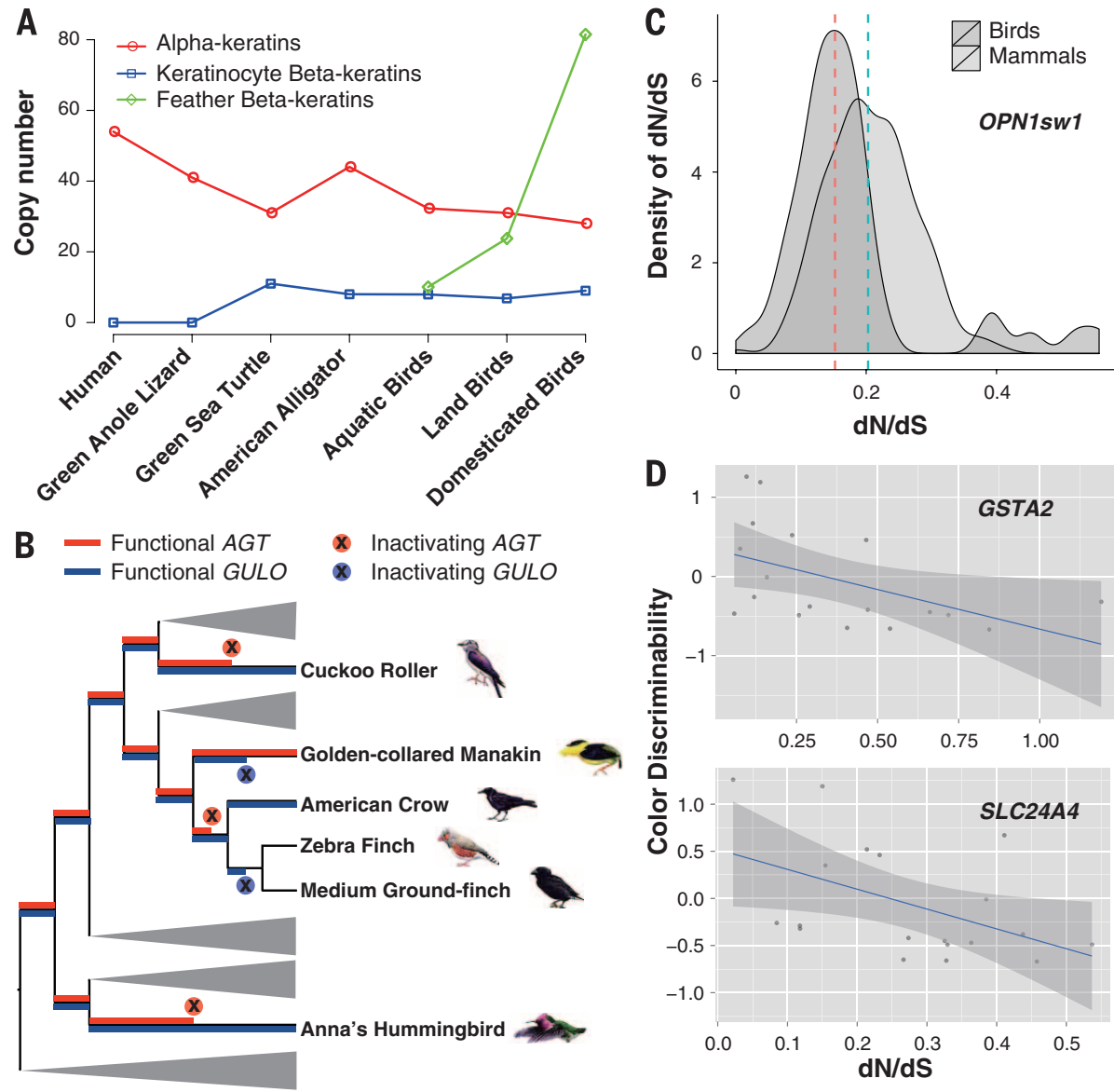

D

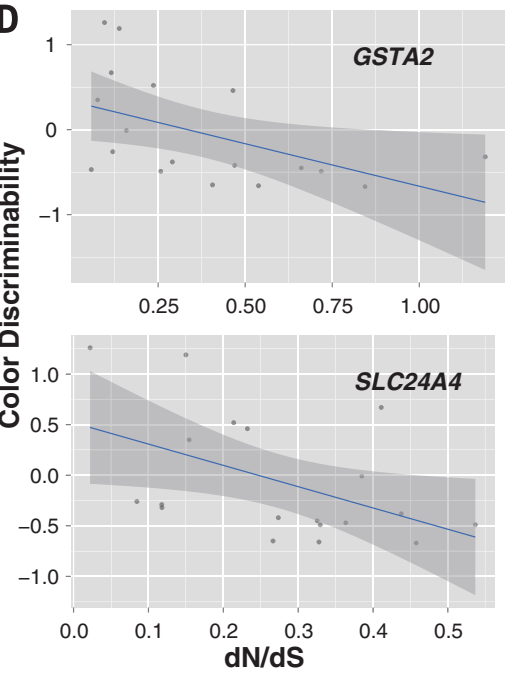

Fig. 6. Genetic changes associated with ecological adaptations. (A) Copy numbers of $\alpha$ - and $\beta$-keratins in humans, reptiles, and birds, including in aquatic birds, land birds, and domesticated birds. Definitions of aquatic and landbirds are provided in (5). (B) Pseudogenization events of the diet-related genes AGTand GULO along the avian phylogeny. (C) Density distribution of $\mathrm{d} N / \mathrm{d} S$ values of the OPN1sw1 gene for mammals (median, 0.21) and birds (median, 0.16). (D) dN/dS values of two plumage color-related genes (GSTA2 and SLC24A4) show negative correlation with the color discriminability values (log transformation applied). The correlation figures with phylogenetically independent contrasts are provided in the supplementary materials. cestral tetrapod set of cones hypothesized to play a role in reproduction and feeding (76). Vertebrate visual opsins are classified into five genes in two families: rhodopsin (RH1) and conopsins (RH2, OPN1sw1, OPN1sw2, and OPN1lw). In most avian genomes, we detected higher numbers of opsin genes than in mammalian genomes, which lacked OPN4x (77), RH2, and either OPN1sw1 (Monotremata) or OPN1sw2 (Theria). All avian genomes contained $\mathrm{RH} 1$ and $\mathrm{RH} 2$, and most high-coverage genomes contained two to three of the remaining three conopsin genes (table S44), supporting that ancestral avian vision is tetrachromatic. Penguins were one of the exceptions, with both species exhibiting only three classes of functional opsins, and thus are trichromatic, which is in line with retinal examination (78). This is likely due to their aquatic lifestyle and is consistent with observations of marine mammals that also appear to have lost one, or even both, cone pigment (or pigments) (76).

Signs of strong positive selection were detected in the branch leading to the passerine group Passerida (represented by the medium groundfinch and zebra finch) (fig. S38), which corroborates that the shift from violet sensitive SWS1 cones in this clade was adaptive (79). Excluding these species, $\mathrm{d} N / \mathrm{d} S$ values for $O P N 1 s w 1$ were lower in birds than in mammals (Fig. 6C). Optimal color discrimination requires an even distribution of spectral sensitivities (80), which is more easily disturbed with an increasing number of cone classes. Hence, stabilizing selection on spectral sensitivity should be stronger in birds than in mammals, and the $\mathrm{d} N / \mathrm{d} S$ values are consistent with this prediction. Besides two transmembrane regions (II and VII) encompassing previously identified spectral tuning amino acids in the SWS1 conopsin, we found markedly positive selection in region IV, strongly suggesting that there is one or more unknown amino acid sites important to spectral tuning of this ultraviolet-sensitive cone (fig. S40).

\section{Sex-related and reproductive traits}

Reproduction-related genes: Unlike other reptiles, almost all birds develop only a single functional ovary, on the left side (81), as a result of the evolutionary loss of the right ovary during the transition from nonavian theropods to birds (82). It has been hypothesized that this loss represents an adaptation to reduce weight during flight (82). We found that two genes related with ovary development (MMP19 and AKR1C3) have been lost in birds. MMP19, a matrix metalloprotease gene, functions during the follicular growth and the ovulation process (83), and the enzyme AKRiC3 catalyzes the conversion of androstenedione to testosterone and has been associated with polycystic ovary syndrome (84).

We analyzed a range of other genes related to reproduction, under the hypothesis that some of them may have been direct targets of the morphological and behavioral adaptations related to sexual selection in birds. Reproduction genes in Drosophila, humans, and marine invertebrates evolve faster than do nonreproduction genes 
(49). We chose 89 genes that may be involved in spermatogenesis (table S46) and six involved in oogenesis (table S47). We found that 19 out of 46 avian species show significantly accelerated evolution (lineage-specific $\mathrm{d} N / \mathrm{d} S$ ratio) of spermatogenesis genes relative to the genomic background (table S48). In contrast, only the carmine bee-eater (Merops nubicoides) and Peking duck showed significantly accelerated evolution in oogenesis genes (table S49). These results suggest that male birds are the dominant targets of sexual selection, which drives rapid evolution of spermatogenesis genes via sperm competition (85).

Plumage color: We investigated the genomics of plumage color, a behaviorally important trait and longstanding example of sexual selection (86). Male birds have frequently evolved extravagant plumage color in response to both malemale competition and female choice $(87,88)$, resulting in remarkable sexual dichromatism. Analysis of 15 genes implicated in avian plumage coloration demonstrated rapid evolutionary rates over the genomic average in 8 of 46 lineages (table S51). This pattern suggests that these genes are evolving under adaptive evolution.

Carotenoids, which are responsible for the bright yellow and red pigments that underlie some of the most conspicuous coloration patterns in vertebrates, unlike melanins can be only acquired through diet and represent trade-offs between coloration and other physiological conditions. We identified a negative correlation between color discriminability and $\mathrm{d} N / \mathrm{d} S$ across birds for the gene GSTA2, which is involved in the binding and deposition of carotenoids and in plumage dichromatism $\left(R^{2}=0.24, P=\right.$ 0.045 , Pearson's test with phylogenetically independent contrasts) (Fig. 6D and fig. S41), and similarly for $S L C 24 A 4$, which is associated with hair color in humans $\left(R^{2}=0.21, P=0.056\right.$, Pearson's test with phylogenetically independent contrasts) (Fig. 6D and fig. S42), suggesting that either diversifying and stabilizing selection or the effect of different population sizes is driving the evolution of plumage color genes.

\section{Discussion and conclusions}

The small genome size of birds with fragmented microchromosomes and reduced repeat transposon activity, in contrast to other vertebrates, has been a static feature in the avian clade for $>100$ million years. Avian genomes consistently contain fewer genes, $\sim 70 \%$ of the number of the human genome, and with one detected exception (downy woodpecker), an extremely reduced fraction of repeat elements. Thus, the ancestral avian lineage has distinctly lost a large number of genes by means of large segmental deletions after their divergence from other extant reptiles. These large genomic sequence deletions appear to be linked to a second defining feature of avian genomes: the putatively ancestral fission of macrochromosomes into a relatively large number of microchromosomes.

Genome conservation in birds-along with regard to sequence, synteny, and chromosomal structure-is remarkable in light of their rapid historical radiation. This is considerably differ- ent from the evolution of mammalian genomes, which although are experiencing a rapid radiation at a similar time, today display richer genome shuffling and variation (89). By comparing the genomes of 48 birds that are constrained within a largely resolved phylogeny, we discovered millions of highly constrained elements comprising $7.5 \%$ of avian genomes. This evolutionary profiling of genomes across $>100$ million years (5) enables their interpretation in a functional genomic context not possible in previous genomic studies restricted to fewer taxa.

The analyses of genome sequences for taxa distributed across the avian phylogeny also explains the rich biodiversity of the avian clade because we identified selective constraints on certain categories of genes in different avian lineages. Convergent evolution also appears to be shaping the evolution of protein-coding genes and their regulatory elements, establishing similar morphological or behavioral features in distantly related bird species, as well as variation in specific gene families that correspond to avian traits and environmental adaptation. We believe that the data and analyses presented here open a new window into the evolution, diversification, and ecological adaptation of tetrapod vertebrates and offers a phylogenomic perspective that helps bridge the chasm between micro- and macroevolution.

\section{REFERENCES AND NOTES}

1. F. Gill, D. Donsker, IOC World Bird List (version 3.5) (2013).

2. L. M. Chiappe, L. M. Witmer, Mesozoic Birds: Above the Heads of Dinosaurs (Univ. California Press, Berkeley, CA, 2002).

3. G. Dyke, G. W. Kaiser, Living Dinosaurs the Evolutionary History of Modern Birds (Wiley-Blackwell, Hoboken, NJ, 2011).

4. A. Feduccia, Trends Ecol. Evol. 18, 172-176 (2003).

5. E. D. Jarvis et al., Science 346, 1320-1331 (2014).

6. B. G. Holt et al., Science 339, 74-78 (2013).

7. W. Jetz, G. H. Thomas, J. B. Joy, K. Hartmann, A. O. Mooers, Nature 491, 444-448 (2012).

8. H. Zeigler, P. E. Marler, in Behavioral Neurobiology of Birdsong December 2002, Hunter College, City University of New York New York, NY, US; this volume is the result of the aforementioned conference which was one of an annual symposium series sponsored by the Hunter College Gene Center. (2004).

9. A. J. Stattersfield, M. J. Crosby, A. J. Long, D. C. Wege, Endemic Bird Areas of the World: Priorities for Conservation. BirdLife Conservation (BirdLife International, Cambridge. UK, 1998)

10. D. J. Alexander, Vet. Microbiol. 74, 3-13 (2000)

11. L. W. Hillier et al., Nature 432, 695-716 (2004).

12. R. A. Dalloul et al., PLOS Biol. 8, e1000475 (2010).

13. W. C. Warren et al., Nature 464, 757-762 (2010).

14. Y. Huang et al., Nat. Genet. 45, 776-783 (2013).

15. X. Zhan et al., Nat. Genet. 45, 563-566 (2013).

16. M. D. Shapiro et al., Science 339, 1063-1067 (2013).

17. E. Dickinson, J. Remsen, The Howard and Moore Complete Checklist of the Birds of the World (Aves Press, Eastbourne, UK, 2013).

18. Materials and methods are available as supplementary materials on Science Online.

19. G. Zhang et al., GigaScience 3, 26 (2014)

20. G. Ganapathy et al., GigaScience 3, 11 (2013).

21. T. R. Gregory, in The Animal Genome Size Database (2005); available at www.genomesize.com.

22. Q. Zhou et al., Science 346, 1246338 (2014).

23. E. S. Lander et al., Nature 409, 860-921 (2001).

24. R. E. Green et al., Science 346, 1254449 (2014)

25. A. L. Hughes, M. K. Hughes, Nature 377, 391 (1995).

26. M. Rho et al., Genome Biol. Evol. 1, 2-12 (2009).

27. S. Morand, R. E. Ricklefs, Trends Genet. 17, 567-568 (2001).

28. E. Waltari, S. V. Edwards, Am. Nat. 160, 539-552 (2002).

29. M. G. Kidwell, Genetica 115, 49-63 (2002).

30. C. Feschotte, E. J. Pritham, Annu. Rev. Genet. 41, 331-368 (2007)
31. M. Lynch, J. S. Conery, J. Struct. Funct. Genomics 3, 35-44 (2003). 32. N. Sela, E. Kim, G. Ast, Genome Biol. 11, R59 (2010).

33. A. Böhne, F. Brunet, D. Galiana-Arnoux, C. Schultheis, J. N. Volff, Chromosome Res. 16, 203-215 (2008).

34. Q. Zhang, S. V. Edwards, Genome Biol. Evol. 4, 1033-1043 (2012).

35. C. L. Organ, A. M. Shedlock, A. Meade, M. Pagel, S. V. Edwards, Nature 446, 180-184 (2007).

36. A. L. Hughes, R. Friedman, Mol. Biol. Evol. 25, 2681-2688 (2008),

37. D. K. Griffin, L. B. W. Robertson, H. G. Tempest, B. M. Skinner Cytogenet. Genome Res. 117, 64-77 (2007)

38. K. R. Bradnam et al., GigaScience 2, 10 (2013).

39. J. Kim et al., Proc. Natl. Acad. Sci. U.S.A. 110, 1785-1790 (2013).

40. F. G. Hoffmann, J. C. Opazo, J. F. Storz, Mol. Biol. Evol. 29 , 303-312 (2012)

41. F. G. Hoffmann, J. C. Opazo, J. F. Storz, Mol. Biol. Evol. 25, 591-602 (2008)

42. M. T. Grispo et al., J. Biol. Chem. 287, 37647-37658 (2012).

43. C. F. Baer, M. M. Miyamoto, D. R. Denver, Nat. Rev. Genet. 8 , 619-631 (2007)

44. C. Venditti, M. Pagel, Trends Ecol. Evol. 25, 14-20 (2010).

45. A. Siepel et al., Genome Res. 15, 1034-1050 (2005).

46. K. Lindblad-Toh et al., Nature 478, 476-482 (2011).

47. B. Nabholz, S. Glémin, N. Galtier, BMC Evol. Biol. 9, 54 (2009).

48. W. F. Lathrop, E. P. Carmichael, D. G. Myles, P. Primakoff, J. Cell Biol. 111. 2939-2949 (1990).

49. W. J. Swanson, V. D. Vacquier, Nat. Rev. Genet. 3, 137-144 (2002)

50. L. A. Pennacchio et al., Nature 444, 499-502 (2006).

51. ENCODE Project Consortium, Nature 489, 57-74 (2012).

52. J. E. Mank, E. Axelsson, H. Ellegren, Genome Res. 17, 618-624 (2007)

53. E. Axelsson, M. T. Webster, N. G. C. Smith, D. W. Burt, H. Ellegren, Genome Res. 15, 120-125 (2005)

54. A. Suh et al., Nat. Commun. 2, 443 (2011).

55. E. D. Jarvis, Ann. N. Y. Acad. Sci. 1016, 749-777 (2004).

56. O. Whitney et al., Science 346, 1256780 (2014).

57. A. R. Pfenning et al., Science 346, 1256846 (2014)

58. H. J. Karten et al., J. Comp. Neurol. 521, 3702-3715 (2013)

59. M. J. Hubisz, K. S. Pollard, A. Siepel, Brief. Bioinform. 12, 41-51 (2011).

60. E. R. Dumont, Proc. Biol. Sci. 277, 2193-2198 (2010).

61. S. C. Gutzwiller, A. Su, P. M. O'Connor, Anat. Rec. 296 867-876 (2013)

62. H. R. Duncker, Respir. Physiol. Neurobiol. 144, 111-124 (2004).

63. J. B. West, R. R. Watson, Z. Fu, Respir. Physiol. Neurobiol. 157 382-390 (2007).

64. F. Gill, Ornithology (W.H. Freeman and Company, New York, 1995)

65. A. R. Haake, G. König, R. H. Sawyer, Dev. Biol. 106, 406-413 (1984).

66. J. Ross-Ibarra, Am. Nat. 163, 105-112 (2004).

67. A. Blirt, G. Bell, Nature 326, 803-805 (1987)

68. A. Louchart, L. Viriot, Trends Ecol. Evol. 26, 663-673 (2011).

69. B. C. Livezey, R. L. Zusi, Zool. J. Linn. Soc. 149, 1-95 (2007).

70. J. Cracraft, Syst. Biol. 31, 35-56 (1982)

71. R. W. Meredith, G. Zhang, M. T. P. Gilbert, E. D. Jarvis, M. S. Springer, Science 346, 1254390 (2014)

72. G. M. Birdsey, J. Lewin, A. A. Cunningham, M. W. Bruford, C. J. Danpure, Mol. Biol. Evol. 21, 632-646 (2004)

73. I. B. Chatterjee, Science 182, 1271-1272 (1973).

74. J. Cui, X. Yuan, L. Wang, G. Jones, S. Zhang, PLOS ONE 6 , e27114 (2011)

75. J. Cui, Y.-H. Pan, Y. Zhang, G. Jones, S. Zhang, Mol. Biol. Evol. 28, 1025-1031 (2011).

76. W. I. L. Davies, S. P. Collin, D. M. Hunt, Mol. Ecol. 21, 3121-3158 (2012)

77. S. S. Pires et al., Proc. Biol. Sci. 274, 2791-2799 (2007).

78. J. K. Bowmaker, G. R. Martin, J. Comp. Physiol. A Neuroethol. Sens. Neural Behav. Physiol. 156, 71-77 (1985).

79. A. Ödeen, O. Håstad, P. Alström, BMC Evol. Biol. 11, 313 (2011)

80. L. Chittka, Naturwissenschaften 83, 136-138 (1996).

81. S. S. Guraya, Ovarian Follicles in Reptiles and Birds (Springer-Verlag, Berlin, Germany, 1989)

82. X. Zheng et al., Nature 495, 507-511 (2013).

83. M. Jo, T. E. Curry Jr., Biol. Reprod. 71, 1796-1806 (2004).

84. M. O. Goodarzi, H. J. Antoine, R. Azziz, J. Clin. Endocrinol. Metab. 92, 2659-2664 (2007)

85. T. R. Birkhead, A. P. Møller, Sperm competition and sexual selection. (Academic Press, 1998).

86. C. Darwin, The Descent of Man, and Selection in Relation to Sex (J. Murray, London, 1871)

87. M. Zuk, J. D. Ligon, R. Thornhill, Anim. Behav. 44, 999-1006 (1992)

88. C. Mateos, J. Carranza, Anim. Behav. 54, 1205-1214 (1997).

89. A. R. Quinlan, I. M. Hall, Trends Genet. 28, 43-53 (2012). 
90. J. F. Storz, J. C. Opazo, F. G. Hoffmann, Mol. Phylogenet. Evol. 66, 469-478 (2013)

91. F. G. Hoffmann, J. F. Storz, T. A. Gorr, J. C. Opazo, Mol. Biol. Evol. 27, 1126-1138 (2010)

\section{ACKNOWLEDGMENTS}

Genome assemblies and annotations of avian genomes in this study are available on the avian phylogenomics website (http://phybirds.genomics.org.cn), GigaDB (http://dx.doi.org/ 10.5524/101000), National Center for Biotechnology Information (NCBI), and ENSEMBL (NCBI and Ensembl accession numbers are provided in table S2). The majority of this study was supported by an internal funding from BGI. In addition, G.Z. was supported by a Marie Curie International Incoming Fellowship grant (300837): M.T.P.G. was supported by a Danish National Research Foundation grant (DNRF94) and a Lundbeck Foundation grant (R52-A5062); C.L. and Q.L. were partially supported by a Danish Council for Independent Research Grant (10-081390): and E.D.J. was supported by the Howard Hughes Medical Institute and NIH Directors Pioneer Award DP10D000448.

\section{The Avian Genome Consortium}

Chen Ye, ${ }^{1}$ Shaoguang Liang, ${ }^{1}$ Zengli Yan, ${ }^{1}$ M. Lisandra Zepeda, ${ }^{2}$ Paula F. Campos, ${ }^{2}$ Amhed Missael Vargas Velazquez, José Alfredo Samaniego, ${ }^{2}$ María Avila-Arcos, ${ }^{2}$ Michael D. Martin, ${ }^{2}$ Ross Barnett, ${ }^{2}$ Angela M. Ribeiro, ${ }^{3}$ Claudio V. Mello, ${ }^{4}$ Peter V. Lovell, ${ }^{4}$ Daniela Almeida, ${ }^{3},{ }^{5}$ Emanuel Maldonado, ${ }^{3}$ Joana Pereira, ${ }^{3}$ Kartik Sunagar, ${ }^{35}$ Siby Philip, ${ }^{35}$ Maria Gloria Dominguez-Bello, ${ }^{6}$ Michael Bunce, ${ }^{7}$ David Lambert, ${ }^{8}$ Robb T. Brumfield, ${ }^{9}$ Frederick H. Sheldon, ${ }^{9}$ Edward C. Holmes, ${ }^{10}$ Paul P. Gardner, ${ }^{11}$ Tammy E. Steeves, ${ }^{11}$ Peter F. Stadler, ${ }^{12}$ Sarah W. Burge, ${ }^{13}$ Eric Lyons, ${ }^{14}$ Jacqueline Smith, ${ }^{15}$ Fiona McCarthy, ${ }^{16}$

Frederique Pitel, ${ }^{17}$ Douglas Rhoads, ${ }^{18}$ David P. Froman ${ }^{19}$

${ }^{1}$ China National GeneBank, BGI-Shenzhen, Shenzhen 518083, China. ${ }^{2}$ Centre for GeoGenetics, Natural History Museum of Denmark, University of Copenhagen, Øster Voldgade 5-7, 1350 Copenhagen, Denmark. ${ }^{3}$ CIMAR/CIIMAR, Centro Interdisciplinar de Investigação Marinha e Ambiental, Universidade do Porto, Rua dos Bragas, 177, 4050-123 Porto, Portugal. ${ }^{4}$ Department of Behavioral Neuroscience Oregon Health \& Science University Portland, OR 97239, USA. ${ }^{5}$ Departamento de Biologia, Faculdade de Ciências, Universidade do Porto, Rua do Campo Alegre, 4169007 Porto, Portugal. ${ }^{6}$ Department of Biology, University of Puerto Rico, Av Ponce de Leon, Rio Piedras Campus, JGD 224, San Juan, PR 009431-3360, USA. ${ }^{7}$ Trace and Environmental DNA laboratony. Department of Environment and Agriculture, Curtin University, Perth, Western Australia 6102, Australia. ${ }^{8}$ Environmental Futures Research Institute, Griffith University, Nathan, Queensland 4121, Australia. ${ }^{9}$ Museum of Natural Science, Louisiana State University, Baton Rouge, LA 70803, USA. ${ }^{10}$ Marie Bashir Institute for Infectious Diseases and Biosecurity, Charles Perkins Centre, School of Biological Sciences and Sydney Medical School, The University of Sydney, Sydney NSW 2006, Australia. ${ }^{11}$ School of Biological Sciences, University of Canterbury, Christchurch 8140, New Zealand. ${ }^{12}$ Bioinformatics Group, Department of Computer Science, and Interdisciplinary Center for Bioinformatics, University of Leipzig, Hrtelstrasse 16-18, D-04107 Leipzig, Germany. ${ }^{13}$ European Molecular Biology Laboratory, European Bioinformatics Institute, Hinxton, Cambridge CB10 1SD, UK. ${ }^{14}$ School of Plant Sciences, BI05 Institute, University of Arizona, Tucson, AZ 85721, USA. ${ }^{15}$ Division of Genetics and Genomics, The Roslin Institute and Royal (Dick) School of Veterinary Studies, The Roslin Institute Building, University of Edinburgh, Easter Bush Campus, Midlothian EH25 9RG, UK. ${ }^{16}$ Department of Veterinary Science and Microbiology. University of Arizona, 1117 E Lowell Street, Post Office Box 210090-0090, Tucson AZ 85721, USA. ${ }^{17}$ Laboratoire de Génétique Cellulaire, INRA Chemin de Borde-Rouge, Auzeville, BP 52627 , 31326 CASTANET-TOLOSAN CEDEX, France. ${ }^{18}$ Department of Biological Sciences, Science and Engineering 601, University of Arkansas, Fayetteville, AR 72701, USA ${ }^{19}$ Department of Animal Sciences, Oregon State University, Corvallis, OR 97331, USA.

\section{SUPPLEMENTARY MATERIALS}

www.sciencemag.org/content/346/6215/1311/suppl/DC1 Supplementary Text

Figs. S1 to S42

Tables S1 to S51

References (92-192)

27 January 2014; accepted 6 November 2014 $10.1126 /$ science. 1251385

\section{Whole-genome analyses resolve early branches in the tree of life of modern birds}

Erich D. Jarvis, ${ }^{1 *}+$ Siavash Mirarab, ${ }^{2 *}$ Andre J. Aberer, ${ }^{3}$ Bo Li, ${ }^{4,5,6}$ Peter Houde, ${ }^{7}$ Cai Li, ${ }^{4,6}$ Simon Y. W. Ho, ${ }^{8}$ Brant C. Faircloth, ${ }^{9,10}$ Benoit Nabholz, ${ }^{11}$ Jason T. Howard, ${ }^{1}$ Alexander Suh, ${ }^{12}$ Claudia C. Weber, ${ }^{12}$ Rute R. da Fonseca, ${ }^{6}$ Jianwen Li, ${ }^{4}$ Fang Zhang, ${ }^{4}$ Hui Li, ${ }^{4}$ Long Zhou, ${ }^{4}$ Nitish Narula, ${ }^{7,13}$ Liang Liu, ${ }^{14}$ Ganesh Ganapathy, ${ }^{1}$ Bastien Boussau, ${ }^{15}$ Md. Shamsuzzoha Bayzid, ${ }^{2}$ Volodymyr Zavidovych, ${ }^{1}$ Sankar Subramanian, ${ }^{16}$ Toni Gabaldón, ${ }^{17,18,19}$ Salvador Capella-Gutiérrez, ${ }^{17,18}$ Jaime Huerta-Cepas, ${ }^{17,18}$ Bhanu Rekepalli, ${ }^{20}$ Kasper Munch, ${ }^{21}$ Mikkel Schierup, ${ }^{21}$ Bent Lindow, ${ }^{6}$ Wesley C. Warren, ${ }^{22}$ David Ray, ${ }^{23,24,25}$ Richard E. Green, ${ }^{26}$ Michael W. Bruford, ${ }^{27}$ Xiangjiang Zhan, ${ }^{27,28}$ Andrew Dixon, ${ }^{29}$ Shengbin $\mathrm{Li},{ }^{30}$ Ning $\mathrm{Li},{ }^{31}$ Yinhua Huang, ${ }^{31}$ Elizabeth P. Derryberry, ${ }^{32,33}$ Mads Frost Bertelsen, ${ }^{34}$ Frederick H. Sheldon, ${ }^{33}$ Robb T. Brumfield, ${ }^{33}$ Claudio V. Mello, ${ }^{35,36}$ Peter V. Lovell, ${ }^{35}$ Morgan Wirthlin, ${ }^{35}$ Maria Paula Cruz Schneider, ${ }^{36,37}$ Francisco Prosdocimi, ${ }^{36,38}$ José Alfredo Samaniego, ${ }^{6}$ Amhed Missael Vargas Velazquez, ${ }^{6}$ Alonzo Alfaro-Núñez, ${ }^{6}$ Paula F. Campos, ${ }^{6}$ Bent Petersen, ${ }^{39}$ Thomas Sicheritz-Ponten, ${ }^{39}$ An Pas, ${ }^{40}$ Tom Bailey, ${ }^{41}$ Paul Scofield, ${ }^{42}$ Michael Bunce, ${ }^{43}$ David M. Lambert, ${ }^{16}$ Qi Zhou, ${ }^{44}$ Polina Perelman, ${ }^{45,46}$ Amy C. Driskell, ${ }^{47}$ Beth Shapiro, ${ }^{26}$ Zijun Xiong, ${ }^{4}$ Yongli Zeng, ${ }^{4}$ Shiping Liu, ${ }^{4}$ Zhenyu Li, ${ }^{4}$ Binghang Liu, ${ }^{4}$ Kui Wu, ${ }^{4}$ Jin Xiao, " Xiong Yinqi, ${ }^{4}$ Qiuemei Zheng, Yong Zhang, ${ }^{4}$ Huanming Yang, ${ }^{48}$ Jian Wang, ${ }^{48}$ Linnea Smeds, ${ }^{12}$ Frank E. Rheindt, ${ }^{49}$ Michael Braun, ${ }^{50}$ Jon Fjeldsa, ${ }^{51}$ Ludovic Orlando, ${ }^{6}$ F. Keith Barker, ${ }^{52}$ Knud Andreas Jønsson, ${ }^{51,53,54}$ Warren Johnson, ${ }^{55}$ Klaus-Peter Koepfli, ${ }^{56}$ Stephen O’Brien, ${ }^{57,58}$ David Haussler, ${ }^{59}$ Oliver A. Ryder, ${ }^{60}$ Carsten Rahbek, ${ }^{51,54}$ Eske Willerslev, ${ }^{6}$ Gary R. Graves, ${ }^{51,61}$ Travis C. Glenn, ${ }^{62}$ John McCormack, ${ }^{63}$ Dave Burt, ${ }^{64}$ Hans Ellegren, ${ }^{12}$ Per Alström, ${ }^{65,66}$ Scott V. Edwards, ${ }^{67}$ Alexandros Stamatakis, ${ }^{3,68}$ David P. Mindell, ${ }^{69}$ Joel Cracraft, ${ }^{70}$ Edward L. Braun, ${ }^{71}$ Tandy Warnow, ${ }^{2,72} \dagger$ Wang Jun, ${ }^{48,73,74,75,76}+$ M. Thomas P. Gilbert, ${ }^{6,43}+$ Guojie Zhang ${ }^{4,77} \dagger$

To better determine the history of modern birds, we performed a genome-scale phylogenetic analysis of 48 species representing all orders of Neoaves using phylogenomic methods created to handle genome-scale data. We recovered a highly resolved tree that confirms previously controversial sister or close relationships. We identified the first divergence in Neoaves, two groups we named Passerea and Columbea, representing independent lineages of diverse and convergently evolved land and water bird species. Among Passerea, we infer the common ancestor of core landbirds to have been an apex predator and confirm independent gains of vocal learning. Among Columbea, we identify pigeons and flamingoes as belonging to sister clades. Even with whole genomes, some of the earliest branches in Neoaves proved challenging to resolve, which was best explained by massive protein-coding sequence convergence and high levels of incomplete lineage sorting that occurred during a rapid radiation after the Cretaceous-Paleogene mass extinction event about 66 million years ago.

\section{I}

he diversification of species is not always gradual but can occur in rapid radiations, especially after major environmental changes $(1,2)$. Paleobiological (3-7) and molecular (8) evidence suggests that such "big bang" radiations occurred for neoavian birds (e.g., songbirds, parrots, pigeons, and others) and placental mammals, representing $95 \%$ of extant avian and mammalian species, after the Cretaceous to Paleogene (K-Pg) mass extinction event about 66 million years ago (Ma). However, other nuclear (9-12) and mitochondrial $(13,14)$ DNA studies propose an earlier, more gradual diversification, beginning within the Cretaceous 80 to $125 \mathrm{Ma}$. This debate is confounded by findings that different data sets (15-19) and analytical methods $(20,21)$ often yield con- trasting species trees. Resolving such timing and phylogenetic relationships is important for comparative genomics, which can inform about human traits and diseases (22).

Recent avian studies based on fragments of 5 [ 5000 base pairs (bp) (8)] and 19 [31,000 bp (17)] genes recovered some relationships inferred from morphological data $(15,23)$ and DNA-DNA hybridization (24), postulated new relationships, and contradicted many others. Consistent with most previous molecular and contemporary morphological studies (15), they divided modern birds (Neornithes) into Palaeognathae (tinamous and flightless ratites), Galloanseres [Galliformes (landfowl) and Anseriformes (waterfowl)], and Neoaves (all other extant birds). Within Neoaves, 

and adaptation

Guojie Zhang et al.

Science 346, 1311 (2014);

DOI: $10.1126 /$ science. 1251385

This copy is for your personal, non-commercial use only.

If you wish to distribute this article to others, you can order high-quality copies for your colleagues, clients, or customers by clicking here.

Permission to republish or repurpose articles or portions of articles can be obtained by following the guidelines here.

The following resources related to this article are available online at www.sciencemag.org (this information is current as of December 11, 2014 ):

Updated information and services, including high-resolution figures, can be found in the online version of this article at:

http://www.sciencemag.org/content/346/6215/1311.full.html

Supporting Online Material can be found at:

http://www.sciencemag.org/content/suppl/2014/12/11/346.6215.1311.DC1.html

A list of selected additional articles on the Science Web sites related to this article can be found at:

http://www.sciencemag.org/content/346/6215/1311.full.html\#related

This article cites 171 articles, 77 of which can be accessed free:

http://www.sciencemag.org/content/346/6215/1311.full.html\#ref-list-1

This article has been cited by 4 articles hosted by HighWire Press; see:

http://www.sciencemag.org/content/346/6215/1311.full.html\#related-urls

This article appears in the following subject collections:

Genetics

http://www.sciencemag.org/cgi/collection/genetics 